\title{
Adipose-derived stem cell conditioned medium attenuates cisplatin-triggered apoptosis in tongue squamous cell carcinoma
}

\author{
YU-JEN CHIU $^{1,2}$, JAI-SING YANG $^{3}$, HAN-SHUI HSU $^{4,5}$, CHI-HAN TSAI $^{1}$ and HSU MA ${ }^{1,2}$ \\ ${ }^{1}$ Division of Plastic and Reconstructive Surgery, Department of Surgery, Taipei Veteran General Hospital; \\ ${ }^{2}$ Department of Surgery, School of Medicine, National Yang Ming University, Taipei; ${ }^{3}$ Department of Medical Research, \\ China Medical University Hospital, China Medical University, Taichung; ${ }^{4}$ Institute of Emergency and \\ Critical Care Medicine, National Yang-Ming University School of Medicine; ${ }^{5}$ Division of Thoracic Surgery, \\ Department of Surgery, Taipei Veterans General Hospital, Taipei, Taiwan, R.O.C.
}

Received May 30, 2017; Accepted November 15, 2017

DOI: $10.3892 /$ or.2017.6126

\begin{abstract}
Autologous fat grafting procedures have noted a markedly increased frequency, not only for cosmetic purposes, but also for deformities after head and neck cancer and breast cancer surgery. Carcinogenesis is always a major concern in cell therapy-related issues. However, there is no literature discussing this issue in head and neck squamous cell carcinoma patients. To evaluate the interaction of tongue cancer cells and adipose-derived stem cells, we performed a series of in vitro experiments. Our results demonstrated that cisplatin significantly reduced the viabilities of SCC-25 and CAL-27 cells in a concentration-dependent manner, but it had low cytotoxicity in cisplatin-resistant CAL-27 (CAR) cells. There was no significant difference in terms of viability among the SCC-25, CAL-27, and CAR cells in the adipose-derived stem cell conditioned medium and control groups. There was also no significant difference in terms of cell migration as determined by wound healing assay of SCC-25, CAL-27, and CAR cells between the adipose-derived stem cell conditioned medium treatment and control treatment. Importantly, the adipose-derived stem cell conditioned medium attenuated cisplatin-triggered cell death in the SCC-25 and CAL-27 cells. Moreover, adipose-derived stem cell conditioned medium
\end{abstract}

Correspondence to: Dr Hsu Ma, Division of Plastic and Reconstructive Surgery, Department of Surgery, Taipei Veterans General Hospital, 19/F, No. 201, Sec. 2, Shipai Road, Beitou, Taipei 11217, Taiwan, R.O.C.

E-mail: sma471124@gmail.com

Abbreviations: ASC-CM, adipose-derived stem cell conditioned medium; BAD, Bcl-2-associated death promoter; CAR, cisplatinresistant CAL-27 cells; DMEM, Dulbecco's modified Eagle's medium; DMSO, dimethyl sulfoxide; EGFR, epidermal growth factor receptor; ERK, extracellular regulated kinases; IGF-1R, insulin-like growth factor 1 receptor; PBS, phosphate-buffered saline; PI, propidium iodide; BCT, breast conservation treatment

Key words: ASC-CM, head and neck squamous cell carcinoma, cisplatin, IGF-1R/AKT/ERK signaling pathway markedly inhibited cisplatin-induced apoptotic cell death (sub-G1 phase) in the CAL-27 cells. Western blot analyses indicated that cisplatin-induced reductions in pro-caspase-3, pro-caspase-9, phospho-BAD, phospho-IGF-1R, phosphoAKT, and phospho-ERK in CAL-27 cells were reversed by adipose-derived stem cell conditioned medium supplement. Taken together, we provide evidence that adipose-derived stem cell conditioned medium protects CAL-27 cells from cisplatin-induced cell death, possibly through upregulation of the IGF-1R/AKT/ERK signaling pathway.

\section{Introduction}

Autologous fat grafts are used to fill soft tissue defects, and were reported to be performed as early as 1893 . With refinements in the technique, autologous fat transplantation has become increasingly popular in cosmetic and reconstructive surgery (1). After liposuction from an area where fat is present in excess, such as the abdomen or thigh, the fat can be grafted for an array of problems, including atrophic and posttraumatic facial deficits, facial rhytids, scarring, and lip and breast augmentation $(2,3)$. Furthermore, adipose tissue has gained significant importance for the complexity of its components and functions over the past decade. The main subcomponents of adipose tissue contain mature adipocytes and a stromal vascular fraction. Recent biotechnological advancements have allowed for the harvesting of adult stem cells from the stromal vascular fraction (3). Using a patient's own stem cells avoids ethical issues associated with the use of human embryonic or bone marrow stem cells, and reduces the chance of tissue rejection. In addition, it is less invasive and much easier to obtain adipose-derived stem cells (ASCs) than performing a bone marrow extraction. ASCs have been proven to efficiently allow stimulation of cell-differentiation, immune-modulation, peripheral nerve repair and angiogenesis (4). In a recent study, ASCs have become powerful tools to improve wound healing and attenuate scar formation by promoting cell migration, angiogenesis and a possible regenerative rather than fibrotic microenvironment at the wound site (5).

Breast asymmetry after breast conservation surgery is challenging for plastic surgeons. Autologous fat grafts with or 
without skin flap reconstruction seems to be a good choice to fill defects and improve cosmetic outcome of breast conservation surgery (6). However, oncological safety is a major concern in cell therapy-related issues; especially in ASCs since they secrete cytokines, chemokines and growth factors, which may promote cancer cell growth, metastasis, and angiogenesis. Multiple studies have discussed the interaction between lung, colon and breast cancer cells, ASCs and fat grafting (7-12). Although autologous fat grafting is not associated with an increased risk of tumor recurrence or distant metastasis of patients with breast cancer in clinical studies $(3,13,14)$, some studies have reported that adipocytes or ASCs may stimulate cancer cell growth or metastasis (9-12).

Patients with head and neck cancer usually have aesthetic and functional problems after excision and reconstructive surgery, such as chin deformity, scar contracture, and drooling. Autologous fat injection is a good method to rebuild postoperative soft tissue volume insufficiency and scar contracture for these patients. Similar to breast cancer patients, carcinogenesis of autologous fat grafting is a critical issue. ASCs may induce tyrosine kinase receptor signaling pathways to promote tumor growth or chemoresistance $(8,15)$. However, the underlying interaction between ASCs and head and neck squamous cell carcinoma remains unclear. In the present study, we aimed to investigate the biological effects and molecular mechanisms of the crosstalk between ASCs and oral cancer cells in vitro.

\section{Materials and methods}

Chemicals and reagents. Dimethyl sulfoxide (DMSO), potassium phosphate, trypan blue, propidium iodide (PI), Triton X-100, Tris-HCl and 3-(4,5-dimethylthiazol-2-yl)2,5-diphenyltetrazolium bromide (MTT) were obtained from Sigma Chemical Co. (St. Louis, MO, USA). Dulbecco's modified Eagle's medium (DMEM), 1.5 mM L-glutamine, 10\% fetal bovine serum (FBS), trypsin-EDTA, penicillin G, and streptomycin were obtained from Gibco BRL (Grand Island, NY, USA). Antibodies against $\beta$-actin (GTX109639, 1:5,000), protein kinase B (Akt; GTX128414, 1:500), phospho-Akt (GTX128414, 1:500), extracellular regulated kinases (ERK1/2; GTX59618, 1:500), phospho-ERK1/2 (GTX59568, 1:500), Bcl-2-associated death promoter (BAD; GTX130108, 1:1,000), phospho-BAD (GTX50136, 1:500), insulin-like growth factor 1 receptor (IGF1R; GTX83064, 1:1,000), phospho-IGF1R (GTX55013, 1:500), epidermal growth factor receptor (EGFR; GTX100448, 1:5,000), phospho-EGFR (GTX61353, 1:500), caspase-3 (GTX110543, 1:1,000), caspase-9 (GTX112888, 1:1,000), and all peroxidase-conjugated secondary antibodies were purchased from Santa Cruz Biotechnology, Inc. (Santa Cruz, CA, USA).

Cell culture. CAL-27 and SCC-25, human tongue squamous cell carcinoma cell lines, were obtained from the Food Industry Research and Development Institute (Hsinchu, Taiwan). Cisplatin-resistant CAL-27 (CAR) cells were kindly provided by Dr Jai-Sing Yang. CAR cells were generated from the CAL-27 cell line by sub-culturing in increasing cisplatin concentrations from 10 to $80 \mu \mathrm{M}$ by 10 cycles of 1 passage, as previously described (16). All cells were cultured in $75 \mathrm{~cm}^{2}$ tissue culture flasks with $90 \%$ DMEM with $1.5 \mathrm{mM}$
L-glutamine adjusted to $1.5 \mathrm{mg} / 1$ sodium bicarbonate, supplemented with $10 \% \mathrm{FBS}$ and $1 \%$ penicillin-streptomycin (100 U/ml penicillin $\mathrm{G}$ and $100 \mathrm{mg} / \mathrm{ml}$ streptomycin), and maintained at $37^{\circ} \mathrm{C}$ in a $5 \% \mathrm{CO}_{2}$ atmosphere.

Adipose tissue-derived stem cell (ASC) preparation. This study was approved by the Institutional Review Board of Taipei Veterans General Hospital (VGHIRB no. 2016-06-005A). The board is organized and operates according to the International Conference on Harmonisation (ICH)/WHO Good Clinical Practice (GCP) and applicable laws and regulations. Briefly, lipo-aspirate was obtained from the liposuction waste of three volunteers. The fat was immersed in phosphate-buffered saline (PBS) solution and centrifuged to remove excessive fluid. The washed fat tissue was mixed with DMEM, collagenase $(1 \mathrm{mg} / \mathrm{ml}), \mathrm{N}$-acetylcysteine $(2 \mathrm{mM})$, and L-ascorbic acid-2 phosphate $(0.2 \mathrm{mM})$ and cultured at $37^{\circ} \mathrm{C}$ for $1 \mathrm{~h}$. After centrifugation to remove excessive collagenase, the pellet was cultured with DMEM (10\% FBS), $N$-acetylcysteine (2 mM), and L-ascorbic acid-2 phosphate $(0.2 \mathrm{mM})$ under $5 \% \mathrm{CO}_{2}$ overnight. The flask was washed with PBS to remove unattached cells, and the medium was changed every other day until confluence. Cells were sub-cultured and characterized by flow cytometry after positive surface staining for CD29 and CD90, but not for CD11B, CD31 or CD45. Before experimental use, ASCs were tested for their ability to differentiate into various mesenchymal lineages, including adipocytes, osteoblasts and chondrocytes (data not shown).

Preparation of ASC conditioned medium (ASC-CM). To prepare ASC-CM, ASCs $\left(1 \times 10^{6}\right)$ were cultured in T75 flasks for 2-3 days. Culture medium was removed at cell confluence, and the cells were washed twice with PBS. Fresh DMEM supplemented with $10 \%$ FBS was added and incubation was carried out for 1 day. After incubation, the culture medium was collected and centrifuged to remove the cells.

Cell viability assay. To evaluate the cytotoxic effect of cisplatin on CAL-27, SCC-25, and CAR cells, an MTT [3-(4,5-dimethylthiazol-2-yl)-2,5-diphenyltetrazolium bromide] assay was used. Briefly, the cells were placed in a 96-well cell culture plate at an initial concentration of $5 \times 10^{4}$ cells $/ \mathrm{ml}$, treated with cisplatin at different concentrations $(5,10,15$ and $20 \mu \mathrm{M})$ or with $0.1 \%$ DMSO for $24 \mathrm{~h}$, and cultured in DMEM or ASC-CM. Each concentration was repeated three times. After a $24-\mathrm{h}$ incubation period, MTT solution $(5 \mathrm{mg} / \mathrm{ml}, 100 \mu \mathrm{l} /$ well $)$ was added to the cells for $4 \mathrm{~h}$. Subsequently, the growth medium was removed, and the formazan crystals formed by oxidation of the MTT solution were dissolved with DMSO in isopropanol and measured spectrophotometrically at $490 \mathrm{~nm}$. The cell survival ratio was expressed as a percentage of the control. Viability assays were performed in triplicate from three independent experiments.

Phase-contrast microscopy of morphological changes. CAL-27 cells were cultured in 6-well plates at a density of $2.5 \times 10^{5}$ cells $/$ well $/ \mathrm{ml}$ before being treated with $20 \mu \mathrm{M}$ of cisplatin or with $0.1 \%$ DMSO for $24 \mathrm{~h}$, and cultured in DMEM or ASC-CM. After treatment for $24 \mathrm{~h}$, morphological changes were determined by a phase-contrast microscope. 
Wound healing assay. CAL-27 and CAR cells were placed in a 6-well tissue culture plate for $24 \mathrm{~h}$ and grown to $80-90 \%$ confluence. Individual wells were scratched with a micropipette tip to create a denuded zone of constant width. Cells were then cultured in serum-low DMEM or ASC-CM for $24 \mathrm{~h}$. Cells were photographed using phase-contrast microscopy (x100). Wound healing assay were performed in triplicate from three independent experiments.

DNA content by flow cytometry. CAL-27 cells $\left(1 \times 10^{5}\right)$ were plated in 24-well plates and cultured in DMEM or ASC-CM with $20 \mu \mathrm{M}$ cisplatin for $24 \mathrm{~h}$. Cells were harvested and then fixed in $70 \%$ ethanol at $24^{\circ} \mathrm{C}$ overnight. Cells were incubated with PI buffer. The distribution of the cell cycle and the sub-G1 population (apoptotic cells) were detected using a flow cytometric method. BD Paint-A-Gate ${ }^{\mathrm{TM}}$ was used for analysis of the flow cytometry (BD Biosciences, San Jose, CA, USA).

Western blot analysis. Briefly, CAL-27 cells were plated in a T75 flask at an initial concentration of $5 \times 10^{6}$ cells, and cultured in DMEM or ASC-CM with $20 \mu \mathrm{M}$ cisplatin for $24 \mathrm{~h}$. The cells were harvested and total protein was collected. Samples were electrophoresed by $10 \%$ sodium dodecyl sulfate-polyacrylamide gel electrophoresis (SDS-PAGE) and transferred onto nitrocellulose membranes (Invitrogen Life Technologies, Carlsbad, CA, USA). The membranes were blocked with PBST solution (0.1\% Tween-20 in PBS) plus 5\% powdered non-fat milk for $1 \mathrm{~h}$, and incubated overnight at $4^{\circ} \mathrm{C}$ with the primary antibody diluted in blocking solution $(0.1 \%$ Tween-20 in PBS plus 5\% powdered non-fat milk). Subsequently, the membranes were washed with PBST three times for $10 \mathrm{~min}$ and incubated with the appropriate alkaline HRP-conjugated secondary IgG antibody (horseradish peroxidase-conjugated goat anti-rabbit and goat anti-mouse) for $1 \mathrm{~h}$ at $24^{\circ} \mathrm{C}$ and then washed three times again. Bands were detected by enhanced chemiluminescence with ECL reagents (Amersham Pharmacia, Buckinghamshire, UK) and exposed on X-OMAT AR film (Eastman Kodak, Rochester, NY, USA). Auto-radiograms were scanned on a UMAX PowerLook Scanner (UMAX Technologies, Fremont, CA, USA) with Photoshop software (Adobe Systems, Seattle, WA, USA). Western blot analysis was performed in triplicate from three independent experiments.

Statistical analysis. All data are expressed as mean \pm SEM from at least three separate experiments. Statistical calculations of the data were performed using a one-way ANOVA. $\mathrm{P}<0.05$ was considered to indicate a statistically significant difference.

Patient data collection. We conducted a retrospective review using data drawn from electronic medical records retained by our medical institution, Taipei Veterans General Hospital. Between January 2000 and December 2014, patients who underwent fat grafting for scar revision after head and neck cancer surgical resection were included in the data collection and analysis.

\section{Results}

Cisplatin reduces the viability of CAL-27 and SCC-25 cells, but does not have an effect on the CAR cells. To determine

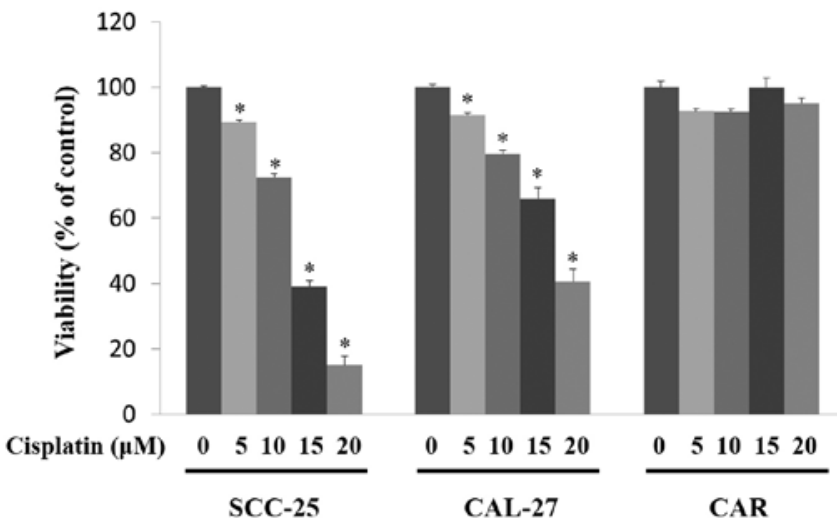

Figure 1. Cisplatin reduces the viability of CAL-27 and SCC-25 cells, but has no effect on cisplatin-resistant CAL-27 (CAR) cells. After treatment with various concentrations of cisplatin for $24 \mathrm{~h}$, the cisplatin treatment significantly decreased the viability of the SCC- 25 and CAL-27 cells in a concentration-dependent manner. There was no cytotoxic effect noted on the cisplatin-treated CAR cells. " $\mathrm{P}<0.05$, statistically significant difference compared to the control group.

the cytotoxic effects of cisplatin on SCC-25, CAL-27 and CAR cells, an MTT assay was performed. As shown in Fig. 1, cisplatin treatment significantly decreased the viability of the SCC-25 and CAL-27 cells in a concentration-dependent manner $(\mathrm{P}<0.05)$. Cisplatin was also found to induce the formation of apoptotic bodies in the SCC-25 and CAL-27 cells after 10-20 $\mu \mathrm{M}$ cisplatin challenge (data not shown). Importantly, there was no cytotoxic effect or morphological characteristic change in the cisplatin-treated CAR cells.

Adipose tissue-derived stem cell conditioned medium (ASC-CM) treatment does not significantly increase human tongue cancer cell viability, invasion and migration abilities. To determine the cell activity of human tongue cancer cells following ASC-CM treatment, viable cells were counted using a hemocytometer after cell culture in DMEM or ASC-CM for $24 \mathrm{~h}$. As shown in Fig. 2A, no significant difference in viability of the SCC-25, CAL-27, and CAR cells was noted between the DMEM and ASC-CM treatment group. We subsequently performed a wound healing assay. As shown in Fig. 2B, our results also failed to reveal a significant difference in the migration ability of the cancer cells between cells cultured in DMEM and ASC-CM.

ASC-CM increases the cell viability of the SCC-25 and CAL-27 cells treated with cisplatin. Phase-contrast microscopy was used to detect the morphological differences between DMEM (control) and ASC-CM groups. As shown in Fig. 3A, CAL-27 cells grew well and spread with a flattened morphology after a 24-h culture. The number of viable cells was decreased and some detached from the surface and contained some debris in the cisplatin-treated CAL-27 cells. However, an increased number of viable cells in the cisplatin-treated CAL-27 cells was observed in the presence of ASC-CM compared to the control group. To detect the growth inhibitory effect of ASC-CM on cisplatin-treated SCC-25 and CAL-27 cells, a cell survival assay by MTT was performed. As shown in Fig. 3, ASC-CM from three different patients increased viable cells in the cisplatintreated SCC-25 (Fig. 3B) and CAL-27 cells (Fig. 3C) ( $<<0.05)$. 

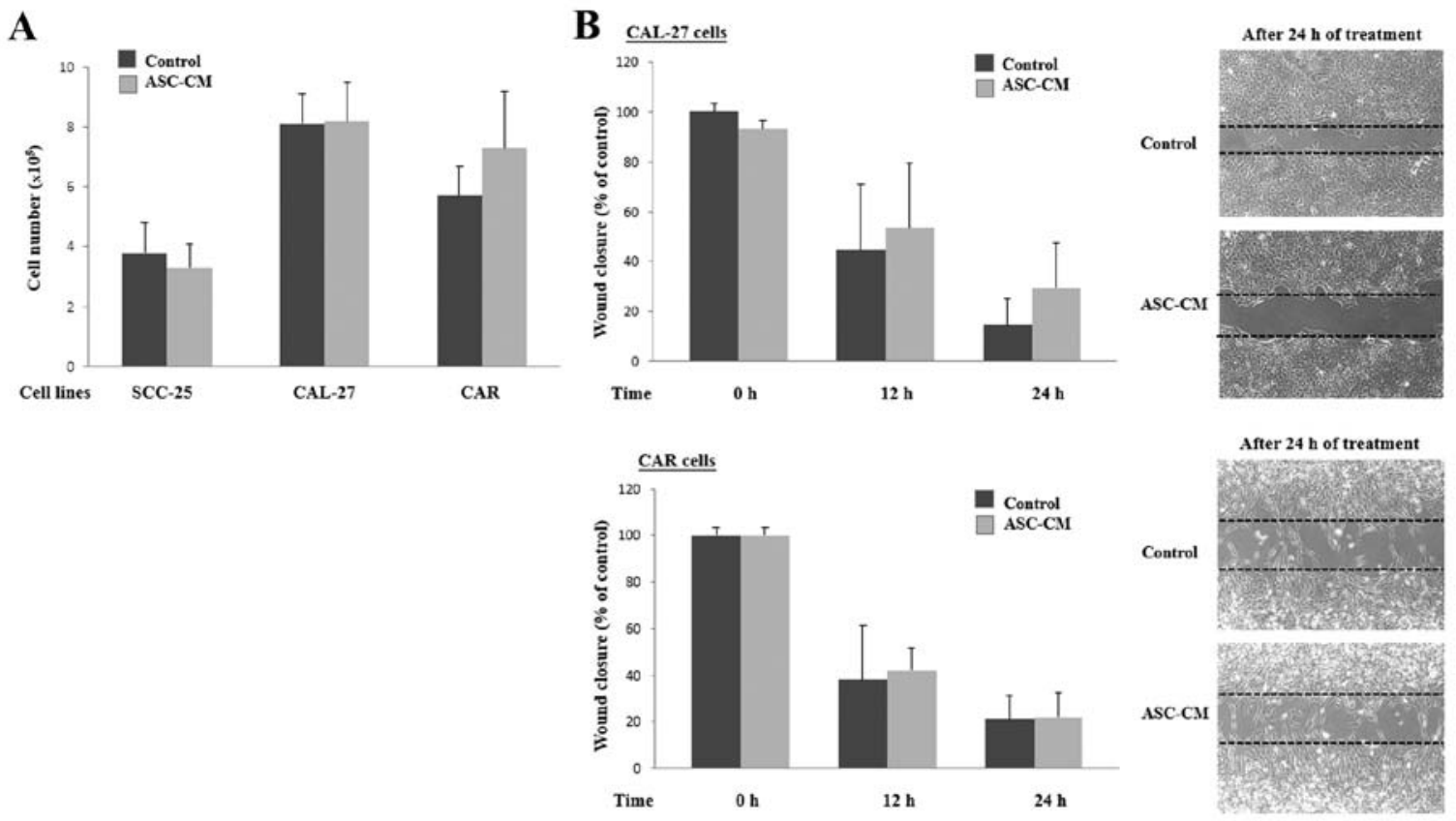

Figure 2. Adipose tissue-derived stem cell condition medium (ASC-CM) treatment did not significantly increase human tongue cancer cell viability and migration ability. Cells were cultured in DMEM (control) or ASC-CM for $24 \mathrm{~h}$. (A) Cell number was not significantly different between tongue cancer cell lines cultured in DMEM and ASC-CM. (B) The wound healing assay demonstrated that CAL-27 and CAR cells cultured in ASC-CM did not exhibit increased migration ability compared to that of cells cultured in control medium (left panel). The width of the scratch after $24 \mathrm{~h}$ of treatment was micro-photographed at magnification x100 (right panel).

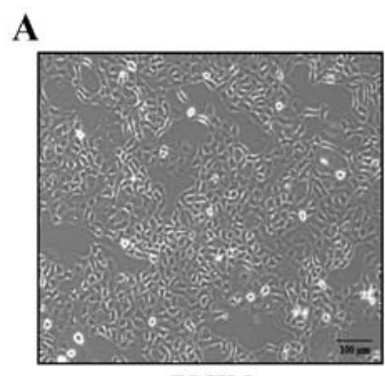

DMEM

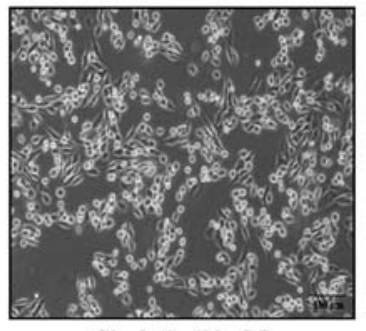

Cisplatin $(20 \mu \mathrm{M})$

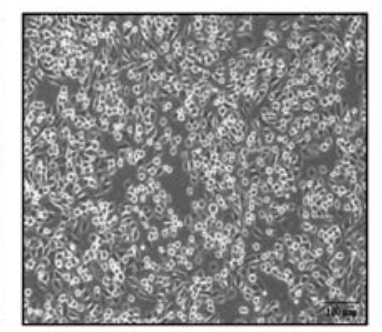

ASC-CM + Cisplatin $(20 \mu \mathrm{M})$

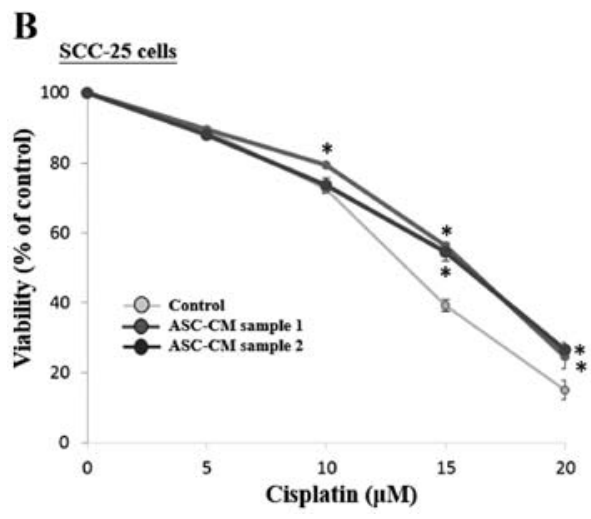

C

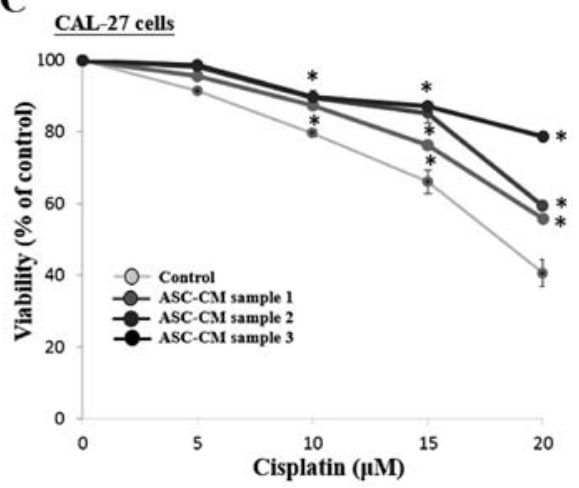

Figure 3. Adipose tissue-derived stem cell condition medium (ASC-CM) increases the cell viability of SCC-25 and CAL 27 cells treated with cisplatin. (A) The cell number was increased in the tongue cancer cells cultured with ASC-CM (x200 magnification). (B and C) MTT assay was performed to detect the growth inhibitory effect of ASC-CM on cisplatin-treated SCC-25 and CAL-27 cells. ASC-CM samples 1, 2 and 3 were obtained from three different patients. SCC-25 (B) and CAL-27 (C) cells cultured with ASC-CM were more resistant to cisplatin treatment compared to cells cultured with control medium. " $\mathrm{P}<0.05$, statistically significant difference compared to the control group.

ASC-CM reduces cisplatin-induced CAL-27 cell apoptosis. To further evaluate the apoptotic process in cisplatin-treated CAL-27 cells under ASC-CM, flow cytometry was used to exam the DNA content of the apoptotic population (sub-G1 phase). As shown in Fig. 4, ASC-CM significantly reduced the sub-G1 phase cell population in the cisplatin-treated CAL-27 
A

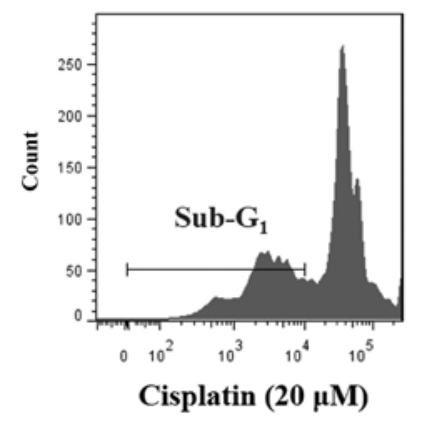

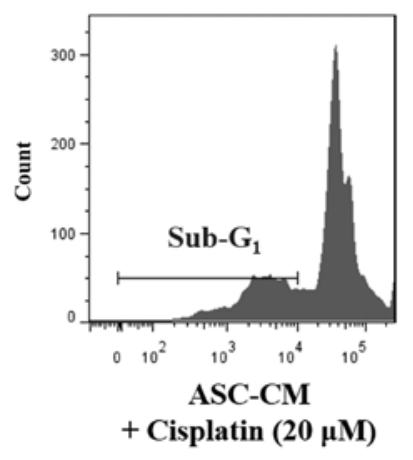

B

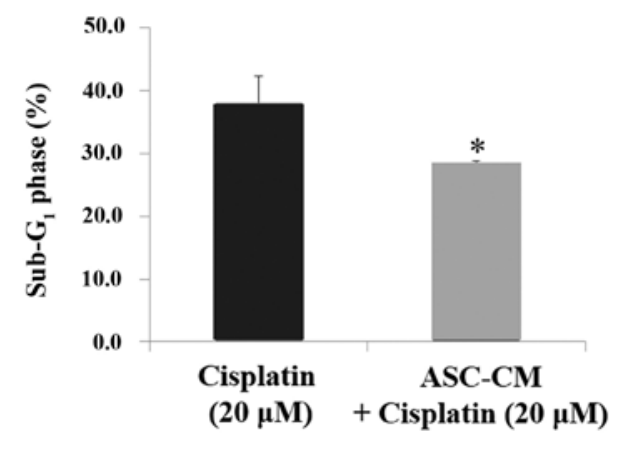

Figure 4. Adipose tissue-derived stem cell condition medium (ASC-CM) reduces cisplatin-induced apoptosis in CAL-27 cells. CAL-27 cells were treated with $20 \mu \mathrm{M}$ cisplatin and cultured in DMEM or ASC-CM for $24 \mathrm{~h}$. (A and B) Flow cytometry showed that the cell population in the sub-G1 phase was significantly decreased in cells cultured with ASC-CM, which suggests that apoptosis was reduced in the CAL-27 cells cultured with ASC-CM after cisplatin treatment, compared to cells cultured in control medium. ${ }^{*} \mathrm{P}<0.05$, statistically significant difference compared to the control group.

A
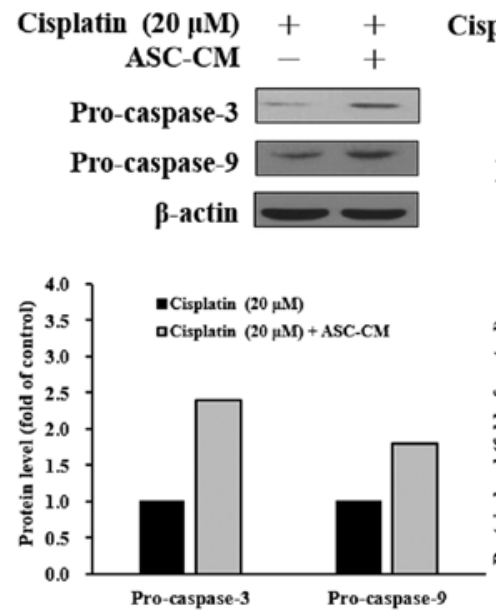

B
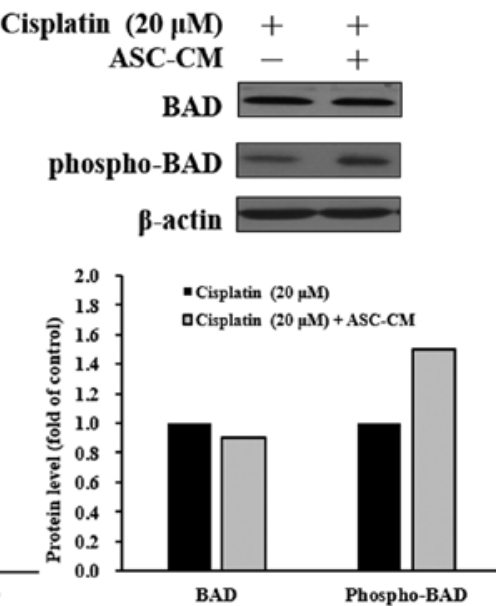

C
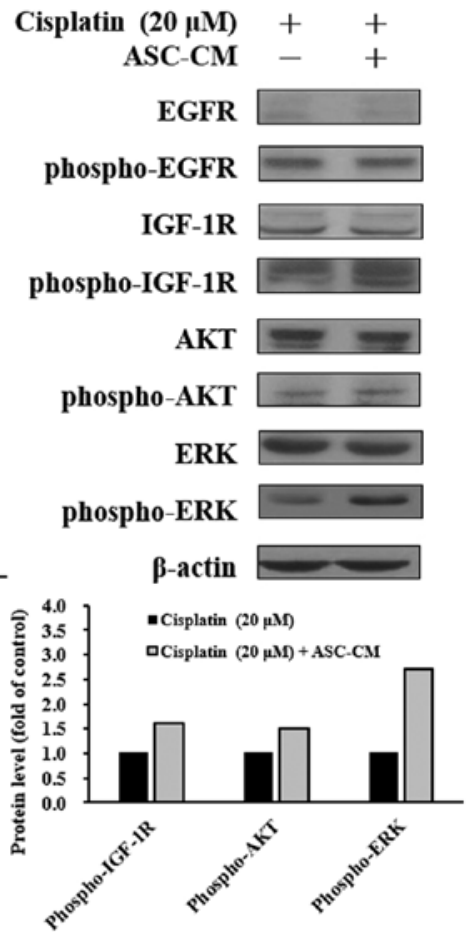

Figure 5. Adipose tissue-derived stem cell condition medium (ASC-CM) modulates caspase-3, caspase-9, and IGF-1R signaling-related protein levels in cisplatin-treated CAL-27 cells. CAL-27 cells were harvested after $20 \mu \mathrm{M}$ cisplatin treatment in DMEM or ASC-CM for $24 \mathrm{~h}$. (A) Western blot analysis showed increased expression of pro-caspase-3 and pro-caspase-9 proteins. (B) Phosphorylation of BAD was also increased in cells cultured with ASC-CM. (C) Increased expression of IGF-1R/AKT-associated proteins including phospho-IGF-1R, phospho-AKT, and phospho-ERK in CAL-27 cells cultured with ASC-CM. These data suggest that ASC-CM attenuates cisplatin-induced apoptosis by phosphorylation of BAD in CAL-27 cells through activation of the IGF-1R/AKT/ERK signaling pathway.

cells $(38.1 \pm 4.2 \%$ in DMEM and $28.4 \pm 0.4 \%$ in ASC-CM, $\mathrm{P}<0.05)$.

ASC-CM modulates caspase-3, caspase-9, and IGF- $1 R$ signaling-related protein levels in cisplatin-treated CAL-27 cells. To elucidate the possible molecular signaling pathways in cisplatin-treated CAL-27 cells with ASC-CM treatment, the protein levels in the tyrosine kinase signaling and apoptotic pathways were evaluated by western blotting. As shown in Fig. 5, ASC-CM caused an increase in protein levels of pro-caspase-3, pro-caspase-9 (Fig. 5A), and phospho-BAD
(Fig. 5B) in the cisplatin-treated CAL-27 cells. To further investigate cell signaling, upstream-associated proteins were also studied. ASC-CM caused an increase in protein expressions of phospho-IGF-1R, phospho-AKT, and phospho-ERK (Fig. 5C). These results suggest that ASC-CM attenuated cisplatin-triggered apoptosis in CAL-27 cells through the IGF-1R/AKT/ERK signaling pathway.

Patients who underwent fat grafting for scar revision after head and neck cancer surgical resection. In the retrospective review, there were 10 patients with head and neck squamous 
Table I. The demographic data of 10 patients with head and neck cancers who underwent fat grafting procedures.a

\begin{tabular}{ccllll}
\hline Patient & Age/sex & \multicolumn{1}{c}{ Cancer type } & \multicolumn{1}{c}{ Staging } & Follow-up & Outcome \\
\hline 1 & $44 / \mathrm{M}$ & Buccal SCC & pT4aN0M0, IVA & 1.9 years & Died from sarcoma \\
2 & $48 / \mathrm{M}$ & Tongue SCC & Data missing & >5 years & Disease-free \\
3 & $46 / \mathrm{M}$ & Lower gingival SCC & pT4aN2bM0,IVA & >5 years & Disease-free \\
4 & $53 / \mathrm{M}$ & Hypopharyngeal SCC & Data missing & >5 years & Disease-free \\
5 & $49 / \mathrm{M}$ & Buccal SCC & pT1N2bM0, IVA & 3.8 years & Disease-free \\
6 & $41 / \mathrm{M}$ & Buccal SCC & pT4aN2bM, IVA & 4.2 years & Disease-free \\
7 & $85 / \mathrm{M}$ & Buccal SCC & pT1N0M0, I & 4.8 years & Disease-free \\
8 & $51 / \mathrm{F}$ & Buccal SCC & pT2N2bM0, IVA & 4.4 years & Disease-free \\
9 & $50 / \mathrm{M}$ & Buccal SCC & pT4aN1M0, IVA & 3.7 years & Disease-free \\
10 & $51 / \mathrm{M}$ & Buccal SCC & pT3N2bM0, IVA & 2.2 years & Disease-free \\
\hline
\end{tabular}

${ }^{a}$ We conducted a retrospective review using data drawn from the electronic medical records retained by our medical institution, Taipei Veterans General Hospital. Between January 2000 and December 2014, 10 patients who underwent fat grafting for scar revision after head and neck cancer surgical resection were included in data collection and analysis. There was no local recurrence in all patients after fat grafting.

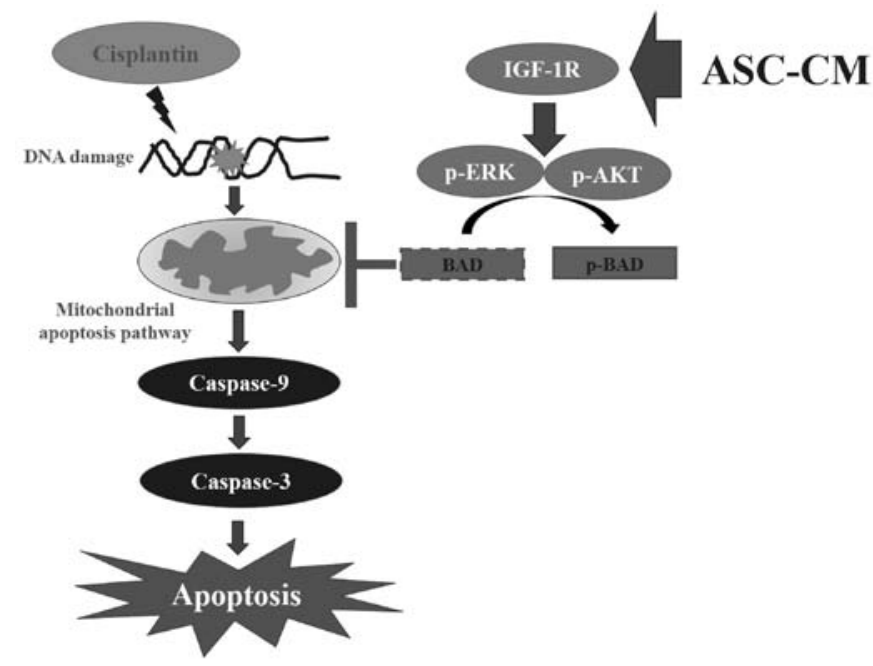

Figure 6. A proposed model illustrating the molecular mechanism and the possible signaling pathways promoted by ASC-CM in cisplatin-treated CAL-27 cells.

cell carcinoma who underwent fat grafting for scar revision after head and neck cancer surgical resection between January 2000 and December 2014. Patient demographics, cancer type, cancer staging after initially surgical resection, follow-up period, and outcome are summarized in Table I. Patient 1 died of radiotherapy-induced sarcoma. There was no local recurrence in all patients during the follow-up period.

\section{Discussion}

With recent advances in techniques and procedures, the frequency of autologous fat grafting has dramatically increased, not only for cosmetic purposes, but also for revision of deformity and reshaping after head and neck cancer and breast cancer surgery. Adipose tissue-derived stem cells (ASCs), a critical component of adipose tissue, has become one of the most popular adult stem cell populations in the field of stem cell research and tissue regeneration (17). However, carcinogenesis is a major concern for cell therapy-related issues. The role of ASCs in clinical applications in breast cancer patients has been disputed intensively in the past decade (14). To the best of our understanding, we investigated the interaction between tongue cancer cells and ASCs, and we found that ASC-CM promoted the chemoresistance of cisplatin-treated CAL-27 and SCC-25 cells.

The clinical effects of the ASC secretome in multiple, biologically relevant scenarios have been widely analyzed and documented, including immunomodulation, angiogenesis, wound healing and tissue regeneration (18). Numerous adipokines have been discovered that could potentially promote breast, lung and colon cancer growth and metastasis $(7-12,19)$. Linkov et al reported that ASC-CM increased endometrial adenocarcinoma proliferation (19). Kim et al demonstrated that ASC-CM enhanced the migration of ovarian cancer cells via activation of the JAK2/STAT3 signaling pathway (20). However, Skelhorne-Gross et al reported that stromal adipocyte PPAR $\gamma$ protects against breast tumorigenesis (21). Zhang et al and Meleshina et al reported that mesenchymal stem cells suppress tumor growth and metastasis by modulating the immune system in mice $(22,23)$. Ryu et al demonstrated that ASC-CM expresses IFN- $\beta$ and suppresses the growth of human breast cancer cells (24). Our results revealed that there was no significant difference in cell growth and cell migration in tongue cancer cells with the addition of condition medium from ASC.

The ASC secretome was also reported to be associated with drug resistance in cancer cells. Nowicka et al reported that adipose stem cells increase ovarian cancer proliferation, migration and chemoresistance (25). Sun et al demonstrated that IGF-1 from ASCs promotes radioresistance of breast cancer cells (15). IGF-1R is a receptor tyrosine kinase, which is involved in tumor progression and promotes cancer cell proliferation, metastasis and chemoresistance (26). Inhibition of IGF-1R could sensitize breast cancer cells to chemotherapy by inhibiting both MAPK and AKT signaling pathways. Abnormal activation of the AKT and ERK signaling cascade stimulates tumor cell growth, proliferation, survival and 
resistance to drug-induced apoptosis (27). Our data demonstrated that ASC-CM inhibited cell death through activation of IGF-1R and ERK/AKT signaling in cisplatin-treated CAL-27 cells (Fig. 5).

Apoptosis is a type of programmed cell death. Two major pathways are involved in apoptotic cell death: the intrinsic (mitochondrial-mediated) and extrinsic (death receptor) pathways. In the intrinsic pathway, regulatory protein is mediated by $\mathrm{Bcl}-2$ family proteins. In apoptotic stimuli, the level of Bax (a pro-apoptotic protein) is increased, which is followed by binding to $\mathrm{Bcl}-2$ (a pro-survival protein) and release of Bax/Bak molecules. Free Bax and Bak lead to cytochrome $c$ release from the mitochondria to the cytoplasm. Released cytochrome $c$ activates the caspase- 9 and caspase- 3 cascade to induce apoptosis $(28,29)$. It was reported that ASC-CM is rich in growth factors, such as IGF-1 and EGF, and these growth factors have an anti-apoptotic function (18). Both ERK and PI-3K pathways provide survival signaling that may neutralize pro-apoptotic Bcl-2 family proteins. ERK induces Bax/Bcl-2 heterodimerization by directly phosphorylating Bcl-2. Regulation of apoptosis by PI-3K is primarily mediated through AKT, which in turn regulates BAD and caspase-9. Phosphorylation of BAD enhances cell survival by inducing cytoplasmic sequestration of Bad through formation of the Bad/14-3-3 protein complex $(21,30)$. In the present study, treatment with condition medium from ASC inhibited cell apoptosis in cisplatin-treated CAL-27 and SCC-25 cells. Furthermore, addition of ASC-CM also resulted in an increase in protein levels of pro-caspase-3, pro-caspase- 9 (Fig. 5A), and phospho-BAD (Fig. 5B) in cisplatin-treated CAL-27 cells. There was a limitation to the results, as we did not routinely perform the western blotting experiments in triplicate.

The ASC secretome could potentially promote tumor initiation and growth, but clinical studies have failed to point out a significant increase in the local recurrence rate of patients who receive autologous fat grafting after breast cancer surgery $(14,18)$. We retrospectively reviewed 10 patients who underwent fat grafting for scar revision after head and neck cancer surgical resection between January 2000 and December 2014 at our institution. Only one patient died of radiation-induced sarcoma. There was no local recurrence in all patients during the follow-up period.

In conclusion, there was no significant difference in cell growth and cell migration between tongue cancer cells with or without addition of condition medium from ASC. Our study revealed that ASC-CM enhanced anti-apoptotic effects through the IGF-1R/AKT/ERK signaling pathway in cisplatin-treated tongue cancer cells. The proposed signal pathways are shown in Fig. 6. This finding provides new insight into the molecular mechanism of condition medium in tongue cancer cells and for its potential therapeutic application. Furthermore, our findings should be taken into consideration when performing fat grafting to the head and neck area. Further investigation to elucidate the function of ASCs in oral cancer is warranted.

\section{Acknowledgements}

This study was supported by a grant from the Taipei Veterans General Hospital, Taipei, Taiwan (V106A-008).

\section{References}

1. Kim J-E and Sykes JM: Hyaluronic acid fillers: History and overview. Facial Plast Surg 27: 523-528, 2011.

2. Delay E, Garson S, Tousson G and Sinna R: Fat injection to the breast: Technique, results, and indications based on 880 procedures over 10 years. Aesthet Surg J 29: 360-376, 2009.

3. Brenelli F, Rietjens M, De Lorenzi F, Pinto-Neto A, Rossetto F, Martella S, Rodrigues JR and Barbalho D: Oncological safety of autologous fat grafting after breast conservative treatment: A prospective evaluation. Breast J 20: 159-165, 2014.

4. Tsuji W, Rubin JP and Marra KG: Adipose-derived stem cells: Implications in tissue regeneration. World J Stem Cells 6: 312-321, 2014.

5. Upadhyay RK: Role of regeneration in tissue repairing and therapies. J Tissue Eng Regen Med 4: 1-30, 2015.

6. Cardoso MJ, Oliveira $\mathrm{H}$ and Cardoso J: Assessing cosmetic results after breast conserving surgery. J Surg Oncol 110: 37-44, 2014.

7. Chen D, Liu S, Ma H, Liang X, Ma H, Yan X, Yang B, Wei J and Liu X: Paracrine factors from adipose-mesenchymal stem cells enhance metastatic capacity through Wnt signaling pathway in a colon cancer cell co-culture model. Cancer Cell Int 15: 42, 2015.

8. Sahin E, Baycu C, Koparal AT, Burukoglu Donmez D and Bektur E: Resveratrol reduces IL-6 and VEGF secretion from co-cultured A549 lung cancer cells and adipose-derived mesenchymal stem cells. Tumour Biol 37: 7573-7582, 2016.

9. Manabe Y, Toda S, Miyazaki K and Sugihara H: Mature adipocytes, but not preadipocytes, promote the growth of breast carcinoma cells in collagen gel matrix culture through cancerstromal cell interactions. J Pathol 201: 221-228, 2003.

10. Iyengar P, Combs TP, Shah SJ, Gouon-Evans V, Pollard JW, Albanese C, Flanagan L, Tenniswood MP, Guha C, Lisanti MP, et al: Adipocyte-secreted factors synergistically promote mammary tumorigenesis through induction of anti-apoptotic transcriptional programs and proto-oncogene stabilization. Oncogene 22: 6408-6423, 2003.

11. Rowan BG, Gimble JM, Sheng M, Anbalagan M, Jones RK, Frazier TP, Asher M, Lacayo EA, Friedlander PL, Kutner R, et al: Human adipose tissue-derived stromal/stem cells promote migration and early metastasis of triple negative breast cancer xenografts. PLoS One 9: e89595, 2014.

12. Chamras H, Bagga D, Elstner E, Setoodeh K, Koeffler HP and Heber D: Preadipocytes stimulate breast cancer cell growth. Nutr Cancer 32: 59-63, 1998.

13. Petit JY, Lohsiriwat V, Clough KB, Sarfati I, Ihrai T, Rietjens M, Veronesi P, Rossetto F, Scevola A and Delay E: The oncologic outcome and immediate surgical complications of lipofilling in breast cancer patients: A multicenter study - Milan-Paris-Lyon experience of 646 lipofilling procedures. Plast Reconstr Surg 128: 341-346, 2011.

14. Charvet HJ, Orbay H, Wong MS and Sahar DE: The oncologic safety of breast fat grafting and contradictions between basic science and clinical studies: A systematic review of the recent literature. Ann Plast Surg 75: 471-479, 2015.

15. Yang HY, Qu RM, Lin XS, Liu TX, Sun QQ, Yang C, Li XH, Lu W, Hu XF, Dai JX, et al: IGF-1 from adipose-derived mesenchymal stem cells promotes radioresistance of breast cancer cells. Asian Pac J Cancer Prev 15: 10115-10119, 2014.

16. Yuan CH, Horng CT, Lee CF, Chiang NN, Tsai FJ, Lu CC, Chiang JH, Hsu YM, Yang JS and Chen FA: Epigallocatechin gallate sensitizes cisplatin-resistant oral cancer CAR cell apoptosis and autophagy through stimulating AKT/STAT3 pathway and suppressing multidrug resistance 1 signaling. Environ Toxicol 32: 845-855, 2017.

17. Raposio E, Caruana G, Bonomini S and Libondi G: A novel and effective strategy for the isolation of adipose-derived stem cells: Minimally manipulated adipose-derived stem cells for more rapid and safe stem cell therapy. Plast Reconstr Surg 133: 1406-1409, 2014.

18. Kapur SK and Katz AJ: Review of the adipose derived stem cell secretome. Biochimie 95: 2222-2228, 2013.

19. Linkov F, Kokai L, Edwards R, Sheikh MA, Freese KE, Marra KG and Rubin JP: The role of adipose-derived stem cells in endometrial cancer proliferation. Scand J Clin Lab Invest Suppl 244: 54-58, 2014.

20. Kim B, Kim HS, Kim S, Haegeman G, Tsang BK, Dhanasekaran DN and Song YS: Adipose stromal cells from visceral and subcutaneous fat facilitate migration of ovarian cancer cells via IL-6/JAK2/STAT3 pathway. Cancer Treat 49: 338-349, 2017. 
21. Skelhorne-Gross G, Reid AL, Apostoli AJ, Di Lena MA Rubino RE, Peterson NT, Schneider M, SenGupta SK, Gonzalez FJ and Nicol CJ: Stromal adipocyte PPAR $\gamma$ protects against breast tumorigenesis. Carcinogenesis 33: 1412-1420, 2012.

22. Zhang L, Su XS, Ye JS, Wang YY, Guan Z and Yin YF: Bone marrow mesenchymal stem cells suppress metastatic tumor development in mouse by modulating immune system. Stem Cell Res Ther 6: 45, 2015.

23. Meleshina AV, Cherkasova EI, Shirmanova MV, Klementieva NV, Kiseleva EV, Snopova LB, Prodanets NN and Zagaynova EV: Influence of mesenchymal stem cells on metastasis development in mice in vivo. Stem Cell Res Ther 6: 15, 2015.

24. Ryu H, Oh J-E, Rhee K-J, Baik SK, Kim J, Kang SJ, Sohn JH, Choi E, Shin HC, Kim YM, et al: Adipose tissue-derived mesenchymal stem cells cultured at high density express IFN- $\beta$ and suppress the growth of MCF-7 human breast cancer cells. Cancer Lett 352: 220-227, 2014.

25. Nowicka A, Marini FC, Solley TN, Elizondo PB, Zhang Y, Sharp HJ, Broaddus R, Kolonin M, Mok SC, Thompson MS, et al: Human omental-derived adipose stem cells increase ovarian cancer proliferation, migration, and chemoresistance. PLoS One 8: e81859, 2013.

26. Chong KY, Subramanian A, Mokbel K and Sharma AK: The prognostic significance of the insulin-like growth factor-1 ligand and receptor expression in breast cancer tissue. J Surg Oncol 104: $228-235,2011$
27. Chang C-H, Lee C-Y, Lu C-C, Tsai FJ, Hsu YM, Tsao JW, Juan YN, Chiu HY, Yang JS and Wang CC: Resveratrol-induced autophagy and apoptosis in cisplatin-resistant human oral cancer CAR cells: A key role of AMPK and Akt/mTOR signaling. Int J Oncol 50: 873-882, 2017.

28. King YA, Chiu YJ, Chen HP, Kuo DH, Lu CC and Yang JS: Endoplasmic reticulum stress contributes to arsenic trioxideinduced intrinsic apoptosis in human umbilical and bone marrow mesenchymal stem cells. Environ Toxicol 31: 314-328, 2016.

29. Lu C-C, Chen H-P, Chiang J-H, Jin YA, Kuo SC, Wu TS, Hour MJ, Yang JS and Chiu YJ: Quinazoline analog HMJ-30 inhibits angiogenesis: Involvement of endothelial cell apoptosis through ROS-JNK-mediated death receptor 5 signaling. Oncol Rep 32: 597-606, 2014.

30. Chiu YJ, Hour MJ, Lu CC, Chung JG, Kuo SC, Huang WW Chen HJ, Jin YA and Yang JS: Novel quinazoline HMJ-30 induces U-2 OS human osteogenic sarcoma cell apoptosis through induction of oxidative stress and upregulation of ATM/ p53 signaling pathway. J Orthop Res 29: 1448-1456, 2011. 